\title{
Method of Carrier Acquisition and Track for HAPS
}

\author{
Mingxiang Guan ${ }^{1}$, Fang Yuan ${ }^{1}$, Xueyuan Wan ${ }^{1}$, Weizhi Zhong ${ }^{2}$ \\ ${ }^{1}$ Department of Electronic Communication Technology, Shenzhen Institute of Information Technology, Shenzhen, China \\ ${ }^{2}$ School of Electronic and Information Technology, Harbin Institute of Technology, Harbin, China \\ E-mail: gmx2020@126.com \\ Received August 30, 2009; revised April 14, 2009; accepted April 20, 2010
}

\begin{abstract}
This paper emphasizes on the characteristics and schemes of carrier acquisition and track in high dynamic and high information-rate situation. Carrier acquisition model is analyzed theoretically and the design principle of carrier acquisition is deduced and described clearly. An algorithm for carrier acquisition in high dynamic and high information-rate situation is provided. This paper also proves the validity of the algorithm and design scheme in high dynamic and high information-rate situation.
\end{abstract}

Keywords: HAPS, High Dynamic and High Information-Rate, Acquisition, Track

\section{Introduction}

Now the HAPS (high altitude platform Station) system has been concentrated on research because of important values in military and application. The HAPS communication system is comprised by the stable HAP as wavelet relay station, controlling devices on the earth, accessing devices and various kinds of wireless users. Recently many research centers including US military are researching the HAPS communication because of many merits such as low expense, fast deployment, little ground devices, convenient retrieve and so on. An information system formed by HAP in stratosphere will be a new generation-system for the wireless communications and the specialized communication system combines the advantages of both terrestrial and satellite communication systems and avoids, to different extents, their disadvantages. Then many countries had spent large manpower and resources to research the HAPS communication system profoundly [1-3].

In the literature $[4,5]$ frequency detector loop was added to the carrier track loop, therefore, the traditional closed-loop technology was substitute with open-loop carrier error estimation technology. A combined method of data phase information and carrier frequency estimation was provided in literature [6] by means of correction of the local numerical control oscillator (NCO) through the frequency and phase error estimation. In the literature $[7,8]$ the author analyzed the frequency and phase error by means of the combined method of self-adaptive filter and flatness technology. In order to implement the carrier acquisition and track in high dynamic and high information-rate situation, the technologies referred to the literatures above can't reach the requirement of the communication system obviously. Therefore this paper provides a new and suitable carrier acquisition and track technology for HAPS communication in high dynamic and high information-rate situation and proved its validity.

\section{Principle of Acquisition and Track}

To comprehend clearly design method and algorithm this paper in high dynamic and high information-rate situation, the design principle of carrier acquisition and track loop is introduced firstly. The base principle is the non-linear disposal of the suppressed carrier QPSK signal, then the carrier is recovered by phase lock loop and referenced carrier is obtained for coherent demodulation.

$$
x(t)=V_{i} \sin \left[\omega_{0} t+\theta_{i}+\theta\right]
$$

Phase shift $\theta_{i}=2 \pi i / N$ may be some discrete value when $l T_{s}<t<(l+1) T_{s}(i=1,2, \ldots, N) ; \theta$ is the phase that need local referenced signal to track. In order to decrease code error resulting from phase offset during the coherent demodulation, therefore phase lock loop is used to track the drift variation when $\theta$ is changed with the time. What phase estimated value will result is a key problem. The maximum $\hat{\theta}$ of likelihood function $p(x / \theta)$ is discussed to be regard as the estimated value of $\theta$. 


$$
\left.\frac{\partial}{\partial \theta} P(x / \theta)\right|_{\theta=\hat{\theta}}=0
$$

In order to resolve $p(x / \theta)$, suppose the input is Equation (3).

$$
x(t)=S(t, \theta)+n(t)
$$

$n(t)$ is narrowband gauss white noise. Supposed that $T_{s}$ is the observed period and $\theta$ is one of the $N$ fixed values, therefore $\theta_{i}$ is fixed value. In the period $T_{s} m$ sampled values corresponding with $x_{1}, x_{2}, \ldots, x_{m}$ random variable compose $m$ dimensions random vector $X$. when $\theta$ is given, the observed random $X$ is a set which is composed by $m$ independent random variables. The union probability density function (pdf) is:

$$
\begin{aligned}
p(X / \theta) & =p\left(x_{1}, x_{2}, \ldots, x_{m} / \theta\right) \\
& =\frac{1}{\left(2 \pi \sigma^{2}\right)^{m / 2}} \exp \left(-\sum_{k=1}^{m} \frac{n_{k}^{2}}{2 \sigma^{2}}\right) \\
& =\frac{1}{\left(2 \pi \sigma^{2}\right)^{m / 2}} \exp \left(-\sum_{k=1}^{m} \frac{\left(x_{k}-S_{k}\right)^{2}}{2 \sigma^{2}}\right)
\end{aligned}
$$

Make sample interval tend to zero. So $m \rightarrow \infty$, Equation (4) is transferred to Equation (5) in the following.

$$
\begin{aligned}
p(X / \theta)= & A_{1} \exp \left\{-\frac{1}{N_{0}} \int_{0}^{T_{s}}[x(t)-S(t, \theta)]^{2} d t\right\} \\
= & A_{1} \exp \left\{-\frac{1}{N_{0}} \int_{0}^{T_{s}} x^{2}(t) d t+\right. \\
& \frac{2}{N_{0}} \int_{0}^{T_{s}} x(t) S(t, \theta) d t- \\
& \left.\frac{1}{N_{0}} \int_{0}^{T_{s}} S^{2}(t, \theta) d t\right\}
\end{aligned}
$$

The first integral in Equation (5) has nothing to $\theta$; the third integral in Equation (5) is signal energy which will not vary with the parameter $\theta$. Therefore the limit of the likelihood function depends on the value of the second integral in Equation (5). $p(x / \theta)$ can rewrite to Equation (6) in the following.

$$
p(X / \theta)=A_{2} \exp \left\{\int_{0}^{T_{s}} x(t) S(t, \theta) d t\right\}
$$

$A_{1}, A_{2}$ is constant respectively.

The value $\theta_{i}=2 \pi i / N$ is carried in the period $T_{s}$ at the same probability and the likelihood function of the $N$ dimension signal can be written as the following:

$$
p(X / \theta)=F \sum_{i=1}^{N} \exp \left\{\frac{V_{i}^{2}}{N_{0}}\left[\xi_{s l}(\theta) \cos \theta_{i}+\xi_{c l}(\theta) \sin \theta_{i}\right]\right\}
$$

Here,

$$
\xi_{s l}(\theta)=\frac{2}{V_{i} T_{s}} \int_{l T_{s}}^{(l+1) T_{s}} x(t) \sin \left(\omega_{0} t+\theta\right) d t
$$

$\xi_{s l}(\theta)=\frac{2}{V_{i} T_{s}} \int_{l T_{s}}^{(l+1) T_{s}} x(t) \sin \left(\omega_{0} t+\theta\right) d t, F$ is a constant.

The solution of the equation $\left.\frac{\partial}{\partial \theta} \ln p(x / \theta)\right|_{\theta=\hat{\theta}}=0$ is $\theta=\hat{\theta}$ which can be obtained from the following equation:

$$
\frac{d}{d \theta} \sum_{i=1}^{N}\left[\xi_{s l}(\theta) \cos \theta_{i}+\xi_{c l}(\theta) \sin \theta_{i}\right]^{N}
$$

The solution $\hat{\theta}$ obtained from the Equation (8) is a non-offset estimated value of carrier phase $\theta$. Carrier component information is switched to carrier component by means of non-linear $N$ power process. When $|\theta-\hat{\theta}| \leq \pi / N$, the Mathematical expectation will be larger than zero if $\theta>\hat{\theta}$; otherwise the Mathematical expectation will be smaller than zero if $\theta<\hat{\theta}$. We can set $N=4$ in the Equation (8) and can get the construction form of QPSK easily. The implementation of carrier link acquisition and track is based on this principle in high dynamic and high information-rate situation for HAPS communication systems.

\section{Method of Acquisition and Track}

The sampled signals are divided into two path signals which will be processed by Quadrature and frequency down-conversion. The output signal of numerical frequency down-conversion is regarded as input signal of differential demodulation and carrier track loop. DQPSK algorithmic diagram of acquisition and track is shown in Figure 1.

The main process of the algorithm is as the following:

The input signal $I_{\Sigma}$ and $Q_{\Sigma}$ after $\mathrm{A} / \mathrm{D}$ sample can be written in digital form: $I_{\Sigma}(k)=A_{\Sigma}(k) \cos \varphi(k)$, $Q_{\Sigma}(k)=A_{\Sigma}(k) \sin \varphi(k)$.

Set $S_{\text {in }}(k)=I_{\Sigma}(k)+j Q_{\Sigma}(K)=A_{\Sigma}(k) e^{-j \varphi(k)}$, therefore:

$$
\begin{aligned}
S_{\text {out }}(k) & =S_{\text {in }}(k) S_{\text {in }}^{*}(k-1) \\
& =A_{\Sigma}(k) A_{\Sigma}(k-1) e^{-j[\phi(k)-\phi(k-1)]}
\end{aligned}
$$

$$
\text { Definition: } \quad \operatorname{Dok}(k)=\operatorname{Re}\left[S_{\text {out }}(k)\right]
$$

$\operatorname{Cross}(k)=\operatorname{Im}\left[S_{\text {out }}(k)\right]$, in the condition of QPSK modulation the equation $A_{\Sigma}(k)=A_{\Sigma}(k-1)=A_{\Sigma} \quad$ will exist. Therefore:

$$
\begin{gathered}
\operatorname{Dok}(k)=A_{\Sigma}^{2} \cos [\varphi(k)-\varphi(k-1)] \\
\operatorname{Cross}(k)=-A_{\Sigma}^{2} \sin [\varphi(k)-\varphi(k-1)]
\end{gathered}
$$

set $\Delta \varphi_{\text {mod }}=\varphi(k)-\varphi(k-1)$, whose phase value is $0, \pi / 2, \pi, 3 \pi / 2$. But only three logical levels ( 0,1 and -1$)$ 


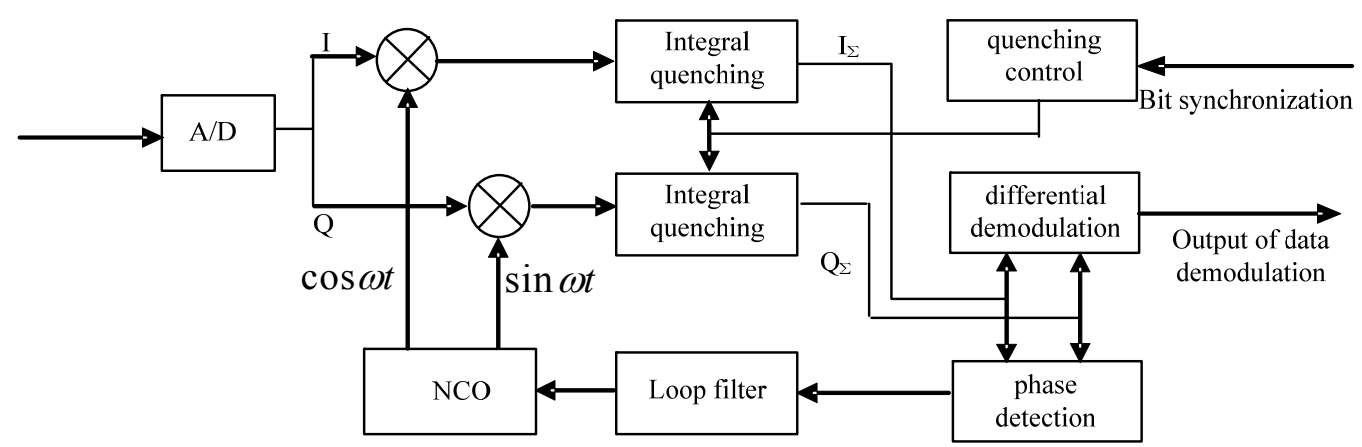

Figure 1. Algorithm of carrier acquisition deriving from DQPSK.

appear when detection is made in Equation (10). Therefore a fixed phase $\pi / 4$ is considered here and the detection method is in the following:

$$
\begin{gathered}
\operatorname{Dok}(k)=A_{\Sigma}^{2} \cos [\varphi(k)-\varphi(k-1)+\pi / 4], \\
\operatorname{Cross}(k)=-A_{\Sigma}^{2} \sin [\varphi(k)-\varphi(k-1)+\pi / 4] .
\end{gathered}
$$

Combine $\Delta \phi_{\text {mod }}=\phi(k)-\phi(k-1)=0, \pi / 2, \pi, 3 \pi / 2$ with the two equations above.

Therefore,

$$
\begin{gathered}
\operatorname{Dok}(k)= \pm \frac{1}{\sqrt{2}} A_{\Sigma}^{2} \\
\operatorname{Cross}(k)= \pm \frac{1}{\sqrt{2}} A_{\Sigma}^{2}
\end{gathered}
$$

Parallel data output after the differential demodulation is corresponding with the four conditions above. In order to synchronize the output signal of NCO and intermediate frequency signal, the frequency of $\mathrm{NCO}$ is adjusted by phase bias of output after differential demodulation. The phase bias control signal is generated by phase detection module. The concrete method is in detail in the following:

Suppose the frequency bias between output of NCO and intermediate frequency signal is $\Delta \omega$ and initial phase bias is $\Delta \varphi$. Therefore the total phase bias is $\Delta \varphi_{e}=\Delta \omega t+\Delta \varphi$. Then:

$$
\begin{gathered}
\operatorname{Dok}(k)=A_{\Sigma}^{2} \cos \left[\Delta \varphi_{\text {mod }}-\Delta \varphi_{e}+\pi / 4\right] \\
\operatorname{Cross}(k)=-A_{\Sigma}^{2} \cos \left[\Delta \varphi_{\text {mod }}-\Delta \varphi_{e}+\pi / 4\right] \\
S_{A F C}=\operatorname{Sign}[\operatorname{Dok}(K)] \operatorname{Cross}(k)-\operatorname{Sign}[\operatorname{Cross}(k)] \operatorname{Dok}(k)
\end{gathered}
$$

is the bias control signal generated by phase detection.

$$
\text { Combine } \quad \Delta \varphi_{\text {mod }}=\varphi(k)-\varphi(k-1)=0, \pi / 2, \pi, 3 \pi / 2
$$
with the equation above.

Therefore:

$$
S_{A F C}=2 A_{\Sigma}^{2} \sin \Delta \varphi_{e}
$$

NCO frequency is controlled by loop filter and $\Delta \varphi_{e}$ will come to zero. Therefore the carrier acquisition and track is implemented in high dynamic and high information-rate situation.

\section{Experiment and Simulation Analysis}

In the design of the carrier acquisition and track loop with high dynamic and high information-rate, the carrier acquisition deriving from DQPSK has a good performance in our experiment. The performance of the algorithm proposed in this paper can't be known directly because of no comparison with other carrier acquisition algorithms. In [5], carrier phase offset, frequency offset and Doppler frequency shift acceleration were simulated in high dynamic situation. So we referred the simulated method in the literature to compare the performance between EKF (Extended Kalman Filter) method and the proposed method in the paper.

With reference to related simulated parameters in [5], the carrier frequency is $10.7 \mathrm{MHz}$, frequency offset is $200 \mathrm{~Hz}$, and initial phase offset is $0.1^{\circ}$. The simulated results are shown in Figure 2. When the frequency is

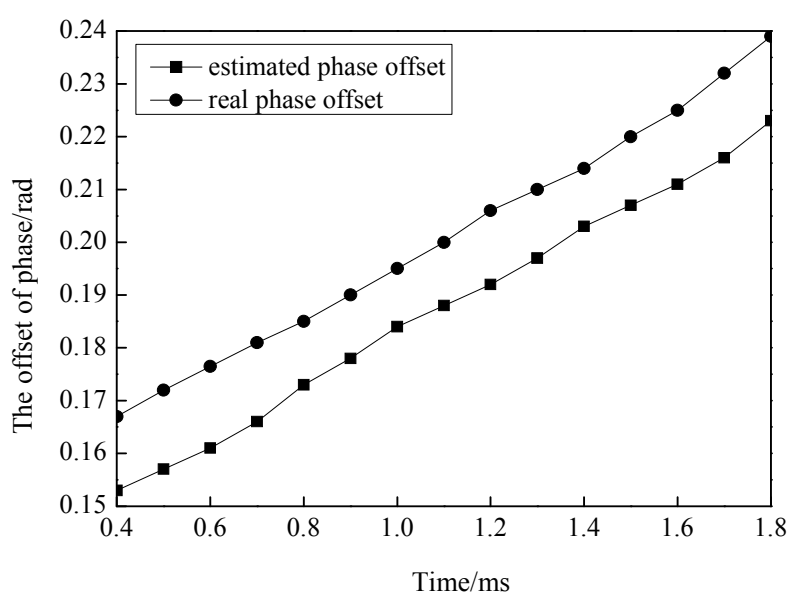

Figure 2. Phase offset estimation of doppler's frequency shift. 


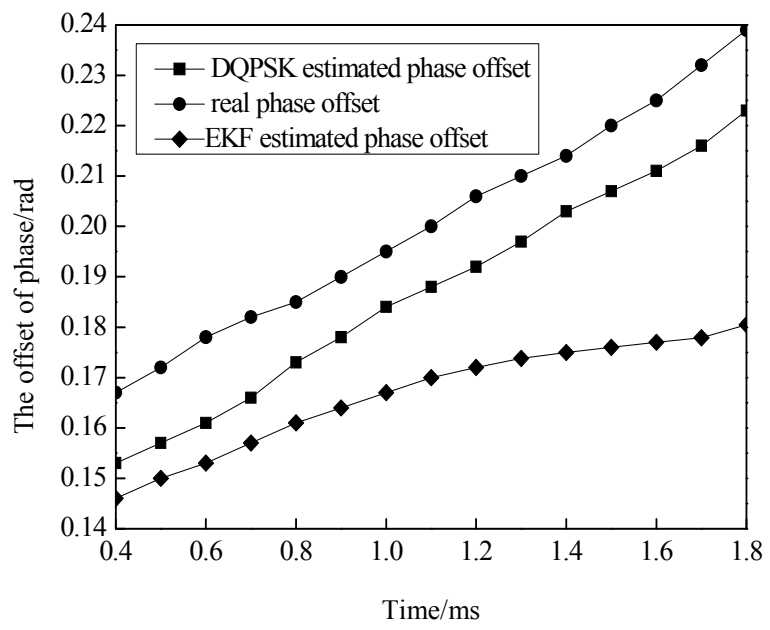

Figure 3. Precision comparison between DQPSK and EFK phase offset estimation.

changed from $200 \mathrm{~Hz}$ to $400 \mathrm{~Hz}$, the real time simulated results are shown in Figure 3. From the Figure 2 DQPSK method can estimate the phase valve accurately. From the Figure 3 phase variation can be tracked less than 20 data time. Therefore the DQPSK method for carrier acquisition is better than EKF method and suitable for high dynamic and high information-rate carrier acquisition and track with a better precision.

\section{Conclusions}

As a branch of the wireless mobile communications with fast development, HAPS communication attracts more and more attention. This paper emphasizes on the characteristics and schemes of carrier acquisition and track in high dynamic and high information-rate situation. Carrier acquisition model is analyzed theoretically and the design principle of carrier acquisition is deduced and described clearly. An algorithm for carrier acquisition in high dynamic and high information-rate situation is provided. This paper also proves the validity of the algorithm and design scheme in high dynamic and high information-rate situation. By comparison with other methods of carrier acquisition and track, the method proposed this paper is suitable with low complexity of hardware and easy to realization in engineering.

\section{Acknowledgements}

This paper is supported by the 2nd doctoral innovation foundation of SZIIT (BC2009015). The author would like to thank Prof. Zhao Honglin to help to design high information-rate intermediate frequency de-spread acquisition and track loop, and Dr. Li Lu for his revision of the text, and the editor and the anonymous reviewers for their contributions that enriched the final paper.

\section{References}

[1] D. Grace, M. H. Capstick, M. Mohorcic and J. Horwath. "Integrating Users into the Wider Broadband Network via High Altitude Platforms," IEEE Wireless Communications, Vol. 12, No. 5, 2005, pp. 98-105.

[2] G. P. White and Y. V. Zakharov, "Data Communications to Trains from High-Altitude Platforms," IEEE Transactions on Vehicular Technology, Vol. 56, No. 4, July 2007, pp. 2253-2266.

[3] E. Cianca, R. Prasad, M. De Sanctis, A. De Luise, M. A. Antonini, D. M. Teotino and M. Ruggieri, "Integrated Satellite-HAP Systems," IEEE Transaction on Communications Magazine, Vol. 43, No. 12, December 2005, pp. 33-39.

[4] B. Wang and H. M. Kwon, 'PN Code Acquisition for DS CDMA Systems Employing Smart Antennas," IEEE Transactions on Wireless Communication, Vol. 2, No. 1, 2003, pp. 108-117.

[5] G. Giunta and F. Benedetto, "Spread-Spectrum Code Acquisition in the Presence of Cell Correlation," IEEE Transactions on Communications, Vol. 55, No. 2, February 2007, pp. 257-261.

[6] H. M. Yu, T. Kang and C. D. Hong, "A New Adaptive Code-Acquisition Algorithm Using Parallel Subfilter Structure," IEEE Transactions on Vehicular Technology, Vol. 55, No. 6, November 2006, pp. 1790-1796.

[7] W. J. Hurd, J. I. Statman and V. A. Vilnrotter, "High Dynamic GPS Receiver Using Maximum Likelihood Estimation and Frequency Tracking," IEEE Transactions on Aerospace and Electronic System, Vol. 23, No. 4, July 1987, pp. 425-437.

[8] E. Karami and M. Shiva, "Blind Multi-Input-MultiOutput Channel Tracking Using Decision-Directed Maximum-Likelihood Estimation," IEEE Transactions on Vehicular Technology, Vol. 56, No. 3, May 2007, pp. 1447-1454. 